This item was submitted to Loughborough's Research Repository by the author.

Items in Figshare are protected by copyright, with all rights reserved, unless otherwise indicated.

\title{
Modelling and simulation of batch pressure filter cycles
}

PLEASE CITE THE PUBLISHED VERSION

PUBLISHER

(C) Elsevier

VERSION

AM (Accepted Manuscript)

LICENCE

CC BY-NC-ND 4.0

REPOSITORY RECORD

Wakeman, Richard J., and E.S. Tarleton. 2009. "Modelling and Simulation of Batch Pressure Filter Cycles". figshare. https://hdl.handle.net/2134/5626. 
This item was submitted to Loughborough's Institutional Repository (https://dspace.lboro.ac.uk/) by the author and is made available under the following Creative Commons Licence conditions.

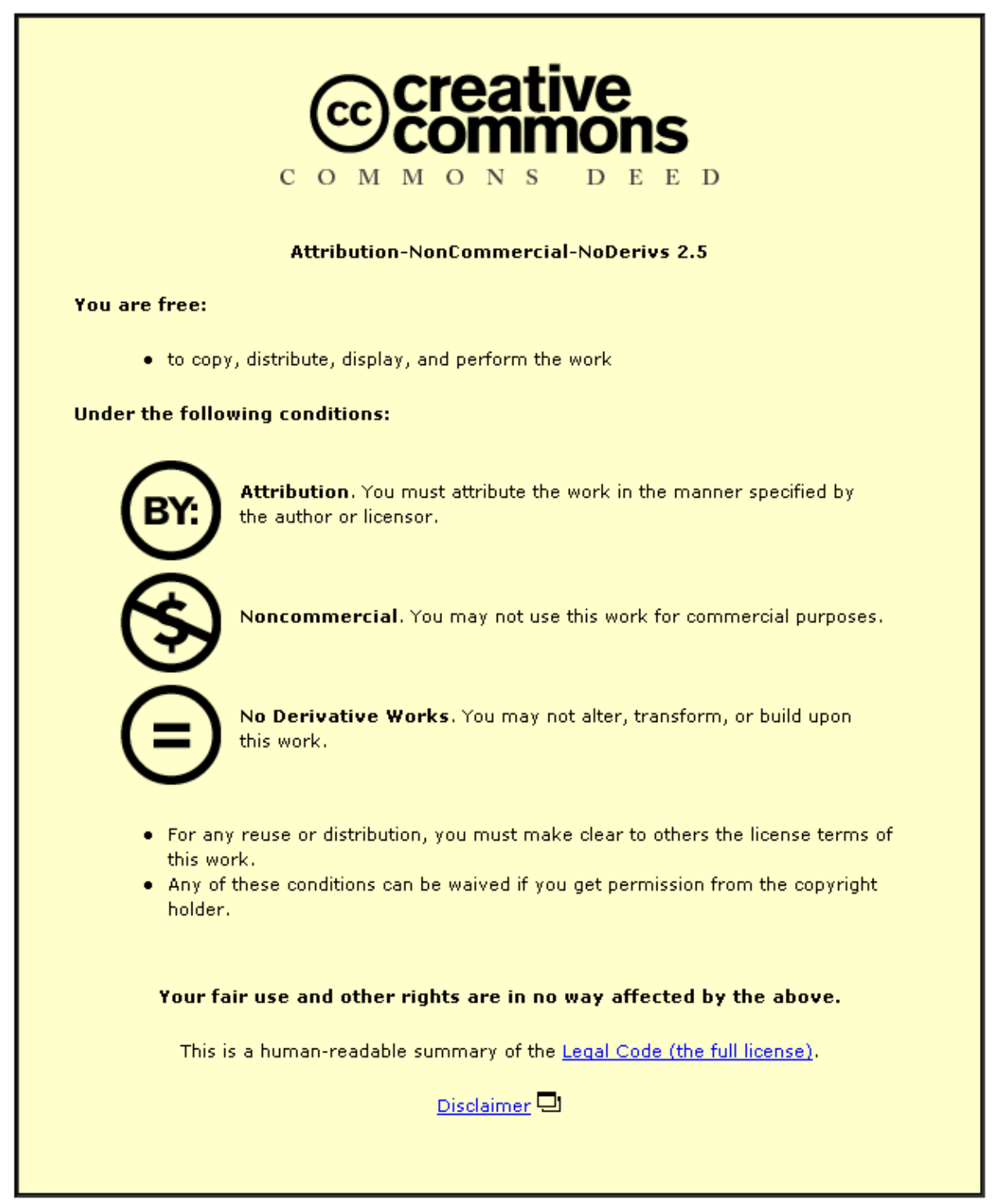

For the full text of this licence, please go to: http://creativecommons.org/licenses/by-nc-nd/2.5/ 


\title{
MODELLING AND SIMULATION OF BATCH PRESSURE FILTER CYCLES
}

\author{
R.J. Wakeman and E.S. Tarleton (e.s.tarleton@lboro.ac.uk) \\ Department of Chemical Engineering, Loughborough University, Loughborough, Leics. LE11 3TU, UK.
}

For the selection, design or optimization of solid/liquid separation equipment it is unusual for an engineer to use fundamental theoretical relationships, and equipment is rarely specified without recourse to extensive laboratory and pilot scale tests. The task of selecting a solid/liquid separator for a given duty has been simplified by the development of rigorous procedures based on theory and practice $^{1-4}$; although filter design procedures are becoming more formalised ${ }^{4}$, software for filter design is generally not available. When a particular type of separator has been identified, the separations technologist is still faced with the prospect of undertaking an extensive test programme to enable scale-up and optimisation of separator size and configuration.

Solid/liquid separation technology, whether it be in the areas of selection, design or optimisation, is best dealt with by interactive computer software. To be most effective the software must incorporate a well chosen mix of algorithms, expert system and input information from the user, be based on sound ideas and proven data from experiment and theory, and maintain the ability to give advice and educate in a congenial fashion so that any psychological barriers to computer use are avoided.

This article describes results from a software package designed to simulate the operation of pressure filters, using data based on the work reported previously ${ }^{5}$, with the aim of demonstrating the general usefulness of the approaches taken. The software lays the foundation for design procedures which may incorporate more comprehensive models for cake formation ${ }^{6-10}$, which are based on more rigorous models of suspension flows and particle deposition, as they become sufficiently practicable. The software utilises industrially proven methodologies that are combined with computer code to produce modular routines capable of modelling cake formation, compression dewatering and washing. The techniques used to describe the operation of the diaphragm filter press are shown and information regarding a typical filter cycle is presented.

\section{THE FILTER CYCLE}

The versatility of the diaphragm filter press allows the range of operating configurations shown in Figure 1. Slurry is fed into the chambers of the press through suitable porting with either a centrifugal, diaphragm, single screw or reciprocating pump ${ }^{11}$ such that filtration can occur at constant rate/variable pressure or variable rate/variable pressure, depending on the type of pump used. Cake is formed simultaneously on the two opposing sides of the filter chamber, with the cake surfaces growing towards each other. If the chambers are not completely filled by cake during this primary filtration stage, then a secondary filtration stage may follow where the suspension remaining between the cakes is filtered at constant pressure by movement of the flexible diaphragm located on one filtering surface. During this stage (ii) process, filtrate is collected from only one drainage surface whilst further cake also forms on the same surface. When sufficient movement of the diaphragm has occurred and the suspension has been filtered for the surfaces of the two cakes to meet as a result of the primary and/or secondary filtration stages, further cake consolidation by the diaphragm (at constant pressure), cake deliquoring by air blowing, and/or cake washing can be undertaken to complete the filter cycle prior to cake discharge. In any single filter cycle, a selection of the stages shown in Figure 1 may be utilised; some stages may be repeated within a cycle, and the order of utilisation can differ between applications. Any simulation methodology must be flexible enough to allow any combination of stages (i) to (v).

Cite paper as: Wakeman R.J. and Tarleton E.S., 1999, Modelling and simulation of batch pressure filter cycles, Filtration and Separation, 36(8), 24-31. DOI: 10.1016/S0015-1882(99)80235-3. Also presented at Software for Filtration and Separation Conference, 1999, The Filtration Society, Birmingham, UK. 
The typical cycle of operations on a tube press is similar to that described above and is illustrated in Figure 2. At the end of the process cake discharge occurs by opening the bottom (in the case of a vertical tube) of the press and applying a reverse pulse of compressed gas inside the cylindrical filter element to release the cake. The model descriptions of each stage in the filter cycle have been tested experimentally, and a comparison of the model predictions against data measured by various authors from full scale, pilot and laboratory filters is given elsewhere ${ }^{4}$.

\section{Stages (i) and (ii): Cake Formation}

It is most common to describe filtration processes by the general filtration equation for the reciprocal volume flow rate of filtrate:

$$
\frac{d t_{f}}{d V_{f}}=\frac{\alpha_{a v} c \mu_{l}}{A_{f}^{2} \Delta p_{f}}+\frac{\mu_{l} R}{A_{f} \Delta p_{f}}
$$

where $\alpha_{a v}$ is the specific resistance of the cake averaged over pressure to the applied pressure difference $\Delta p_{f}, \mu_{l}$ is the filtrate viscosity, $A_{f}$ is the filter area, $R$ is the resistance of the filter medium, and the effective concentration of solids in the feed, $c$, is given by:

$$
c=\frac{\rho M_{s}}{\left(1-m_{a v} M_{s}\right)}
$$

where $\rho$ is the density of the filtrate, $M_{S}$ is the mass fraction of solids in the feed suspension, and $m_{a v}$ is the ratio of the mass of the wet cake to the mass of the dry cake. In the most general sense, both the pressure and rate can vary and it is necessary to impose the pump characteristics on the governing equations. The equations that enable calculation of the filtrate volume, filtration time and cake thickness are summarised for various filter designs and pumping conditions in Table 1.

To account for the potential compressibility of the cake, for practical purposes it is necessary to relate the specific resistance $(\alpha)$ and voids ratio $(e)$ of the filter cake (evaluated at a particular pressure) to the solids compressive pressure through so-called constitutive equations ${ }^{12,13}$ similar to:

$$
\begin{aligned}
& \alpha=\alpha_{0} p_{s}^{n} \text { or } \alpha=\alpha_{0}\left(1+p_{s}\right)^{n} \\
& e=e_{0}-b \log \left(1+p_{s}\right)
\end{aligned}
$$

Equations (3) and (4) are used with equations (5) to (16) and mass balances on the solids in the cake to yield the filtrate volume produced, the filtration time and the cake thickness. This data forms the starting point for the next stage in the filter cycle.

When unfiltered suspension remains in the filter chamber(s) a secondary filtration phase may follow the initial primary filtration. If the secondary phase occurs, then further filtration takes place at constant pressure (with the pressure applied by the diaphragm rather than the pump), with filtrate draining through one filtration surface in each chamber. The cake formation equation is modified to account for the effective increase in resistance to filtration due to the presence of a filter cake on the filter medium at the start of the secondary filtration: 


$$
\frac{t_{f}-\left(t_{f}\right)_{t r}}{V_{f}-\left(V_{f}\right)_{t r}}=\frac{\alpha_{a v} c \mu_{l}}{2 A^{2} \Delta p_{f}}\left(V_{f}+\left(V_{f}\right)_{t r}\right)+\frac{\mu_{l}}{A \Delta p_{f}}\left(\frac{\alpha_{a v} c\left(V_{f}\right)_{t r}}{A}+R\right)
$$

where the filter area effective during secondary filtration $(A)$ equals $A_{f}$ in a plate and frame press and $0.5 A_{f}$ in a diaphragm press. The analogous equation for a tube press is:

$$
\frac{t_{f}-\left(t_{f}\right)_{t r}}{V_{f}-\left(V_{f}\right)_{t r}}=\left(\frac{K_{1}}{2}\left(V_{f}+\left(V_{f}\right)_{t r}\right)+K_{3}\right)+\frac{2 c}{r_{0} \rho_{c}\left(A_{f}\right)_{t r}}\left(\frac{K_{1}}{3}\left(V_{f}^{2}+V_{f}\left(V_{f}\right)_{t r}+\left(V_{f}\right)_{t r}^{2}\right)+\frac{K_{3}}{2}\left(V_{f}+\left(V_{f}\right)_{t r}\right)\right)
$$

where

$$
K_{3}=\frac{\mu_{l}}{A_{f 0} \Delta p_{f}}\left(R+\frac{\alpha_{a v} c\left(V_{f}\right)_{t r}}{\left(A_{f}\right)_{t r}}\right)
$$

\section{Stage (iii): Cake Consolidation}

Cake consolidation is performed at constant pressure through use of a diaphragm and is analysed via an empirical relationship relating the consolidation ratio:

$$
U_{c}=\frac{L_{0}-L}{L_{0}-L_{\infty}}
$$

to a dimensionless consolidation time defined by:

$$
T_{c}=\frac{n_{1}^{2} C_{c} t_{c}}{\omega_{o}^{2}}
$$

such that

$$
U_{c}=\frac{\sqrt{4 T_{c} / \pi}}{\left(1+\left(4 T_{c} / \pi\right)^{v}\right)^{2 v}}
$$

where $C_{c}$ is the consolidation coefficient and $v$ the consolidation behaviour index. Values for $v$ and the other scale-up constants in the constitutive equations for the consolidation phase can be obtained through laboratory tests with, for instance, a leaf filter or a piston press (as previously described ${ }^{4,5}$ ) and related through equations (19) to (21) to give expressions for cake thickness and filtrate volume during the consolidation period(s).

Consolidation is assumed to end when the flow rate of liquid from the filter falls below a predetermined, usually uneconomic, level.

\section{Stage (iv) Cake Deliquoring: (by air blowing)}

The deliquoring phase is analysed through a combination of empirical and theoretical relations. These facilitate the calculation of the time required to achieve a given filter cake moisture content and the air 
flow rate through the cake during the deliquoring period, and have been described in detail ${ }^{14-16}$. Two parameters of prime importance in deliquoring are the pressure required to initiate deliquoring, $p_{b}$, and the irreducible saturation of the filter cake, $S_{\infty}$. Although these should be measured experimentally, the former can be determined with sufficient accuracy from:

$$
p_{b}=\frac{4.6\left(1-\varepsilon_{a v}\right) \sigma}{\varepsilon_{a v} x}
$$

Correlations exist (for relatively large, granular materials) which allow estimation of $S_{\infty}$, for example:

$$
\begin{aligned}
& N_{c a p}=\frac{\varepsilon_{a v}^{3} x^{2}\left(\rho g L+\Delta p_{d}\right)}{\left(1-\varepsilon_{a v}\right)^{2} L \sigma} \\
& S_{\infty}=0.155\left(1+0.031 N_{c a p}^{-0.49}\right)
\end{aligned}
$$

Similar correlations are not available for fine particle cakes, and the above should not be used if alternative data are available. With data for $p_{b}$ and $S_{\infty}$ available, and assuming that no deliquoring of the cake occurs through evaporation of the mother liquor and that the displacing fluid is saturated instantly as it enters the cake, a dimensionless saturation $\left(S_{R}\right.$, termed the reduced saturation) can be related empirically to a dimensionless time, $\theta$, by ${ }^{4}$ :

$$
\begin{aligned}
& S_{R}=\frac{S-S_{\infty}}{1-S_{\infty}} \\
& \theta=\frac{p_{b}}{\Delta p_{d}}\left(0.926\left(\frac{1}{S_{R}}-1\right)\right)^{1.136}
\end{aligned}
$$

for $0.096 \leq\left(\frac{\theta \Delta p_{d}}{p_{b}}\right) \leq 1.915$

$\theta=\frac{p_{b}}{\Delta p_{d}}\left(0.685\left(\frac{1}{S_{R}}-1\right)\right)^{2.083}$

for $1.915 \leq\left(\frac{\theta \Delta p_{d}}{p_{b}}\right) \leq 204$

The deliquoring time $\left(t_{d}\right)$ is then estimated from:

$$
t_{d}=\frac{\theta \varepsilon_{a v} \mu_{l} L^{2}\left(1-S_{\infty}\right)}{\Delta p_{d} k_{a v}}
$$


Allowing for losses in the feed pipes to the filter and the effects of plant elevation, the air flow rate required to achieve the desired moisture content can be calculated ${ }^{4,15-17}$, and the gas or air blower sized accordingly.

\section{(v) Cake Washing: (by displacement)}

The technique used in the analysis of washing phase(s) employs the dispersion model ${ }^{4,17-21}$ to interpret the rates of solute mass transfer from the voids in the filter cake. Use of the model requires a superficial wash velocity, which is estimated from the modified Darcy equation:

$$
u_{0}=\frac{\Delta p_{w}}{\mu_{w}\left(\alpha_{a v} \rho_{s} L\left(1-\varepsilon_{a v}\right)+R\right)}
$$

and the product of the Reynolds and Schmidt numbers, calculated from:

$$
\operatorname{ReSc}=\frac{u_{0} x}{\varepsilon_{a v} D}
$$

The dispersion coefficient varies with the filter cake thickness ${ }^{20}$, and is calculated from:

$$
\begin{aligned}
\frac{D_{L}}{D} & =0.707+55(\operatorname{ReSc})^{0.96} \text { for } 0 \leq L \leq 0.1 \mathrm{~m} \\
& =0.707+1.75(\operatorname{ReSc}) \text { for } L>0.1 \mathrm{~m}
\end{aligned}
$$

The dispersion number is then given by:

$$
D_{n}=\frac{(\operatorname{ReSc}) L}{x \frac{D_{L}}{D}}
$$

If the degree of washing is specified in terms of the required removal of solute, the number of wash ratios used in the washing can be calculated using design charts derived from solutions of the differential equations describing solute dispersion in filter cakes ${ }^{18-21}$. With a knowledge of the number of wash ratios to be applied, the washing time $\left(t_{w}\right)$, effluent solute concentration $(\phi)$ and fractional solute recovery $(F)$ can be estimated. Allowances are made for cake saturation at the start of washing, and for the scale and type of filter being used for the separation ${ }^{17,18}$.

\section{FILTER CYCLE SIMULATION}

The above methodologies enable flexible simulations to be developed. When suitably applied these can model most combinations of cake formation, consolidation, dewatering and washing to allow preliminary sizing and scale-up of equipment to be performed. Although the example of a diaphragm press has been chosen below, the procedures can be readily adapted to model the performance of other filter types. Figure 3 illustrates in simplified form the basic steps in a filter cycle simulation, based on software developed by the authors. At the start the filter type and the required cycle phases are interactively defined by the user along with the file names where the data are to be stored on 
computer disk. The information relevant to each phase is also interactively defined and the magnitude of the numbers entered is checked by the software as much as this is possible. The cycle calculations are then performed for the predefined sequential phases to give the necessary data that describe the filter operation. The results data are displayed either graphically or in tabular form on the computer screen and printed and/or saved to computer disk as appropriate. The data shown below illustrate how information associated with the entire filter cycle can be produced for a set of typical operating conditions through software simulation techniques.

\section{Example of a Computer Simulation}

A diaphragm filter press has been identified as suitable for producing a nominal 5 te of dry particulate solids from an $8 \% \mathrm{w} / \mathrm{w}$ slurry. Following the formation stage(s), the homogeneity of the cake is to be improved through consolidation with the diaphragm. The consolidation must be no longer than the cake formation time. The resultant compact is then to be washed until the solute concentration in the cake liquids is reduced from $30 \mathrm{~kg} \mathrm{~m}^{-3}$ to a total mass of solute in the cake of $1 \mathrm{~kg}$. A deliquoring period then reduces the moisture content of the cakes to $20 \%$ for discharge. The feed is delivered to the filter press via a centrifugal pump whose characteristics are shown in Table 2. The feed suspension characteristics have been determined experimentally and are shown in Table 3 together with suggested operational parameters for the filter.

It is known in practice that greater productivity is obtained from filters if thinner cakes are formed. Using the above data as a basis for calculations and maintaining the values of all other parameters constant, the effects of cake thickness can be examined by performing a number of simulations where the maximum cake thickness (per filter surface) at the end of the filtration is varied over the range from 10 to $40 \mathrm{~mm}$. In all cases where the cake thickness remains below $40 \mathrm{~mm}$ both primary (pump driven) and secondary (diaphragm driven) filtration phases occur. When $40 \mathrm{~mm}$ cakes are formed the cakes meet in the middle of each filter chamber at the end of the primary filtration stage. Examples of the many effects of formed cake thickness are shown in Figures 4 to 11.

Although it may be intuitive that thicker cakes will tend to form at slower rates dependent on the shape of the pump curve (Figure 4), and the pressure drop across thicker cakes is greater (Figure 6), the other effects of limiting or allowing further cake growth in the diaphragm press are not quite so obvious a priori because of the interactions between so many variables.

A larger pressure drop across a compressible cake could potentially cause more compaction, however, the time taken to compact a thicker cake is considerably greater than that needed to compact a thinner one (Figures 7 and 8). Hence the filtrate flux from a thicker cake tends to be rather lower than for a thinner cake. An uneconomic flux is therefore reached when the cake is in a less consolidated state, leaving the 'thicker' cake with a more open structure; in the particular examples shown, during the consolidation phase the thickness of the $40 \mathrm{~mm}$ cake (per filter surface) is reduced by $\sim 14 \%$ compared to the $\sim 54 \%$ of the $10 \mathrm{~mm}$ cake. The more open structure of the final cake is reflected in higher wash liquor rates (Figure 5).

When thicker cakes are formed under the chosen conditions the washing efficiency is marginally improved as the number of required wash ratios reduces from 1.34 (10 mm cake) to 1.04 (40 mm cake), however, the time required to perform a washing operation is significantly increased (Figure 10). Thicker cakes also significantly affect deliquoring and for the given example this has perhaps the greatest impact on the filter cycle. Deliquoring times are more than two orders of magnitude higher with $40 \mathrm{~mm}$ cakes compared to cakes formed at $10 \mathrm{~mm}$ and design air rates are also raised significantly (Figure 11).

Cite paper as: Wakeman R.J. and Tarleton E.S., 1999, Modelling and simulation of batch pressure filter cycles, Filtration and Separation, 36(8), 24-31. DOI: 10.1016/S0015-1882(99)80235-3. Also presented at Software for Filtration and Separation Conference, 1999, The Filtration Society, Birmingham, UK. 
The simulations, Figures 4 to 11 and the summary data shown in Table 4, suggest that increasing the cake thickness causes a number of changes in the cycle which impact on the economics of the separation.

The main factors are:

a. The extension of the cycle time which, for the particular examples chosen, has greatest effect in lengthening of the deliquoring phase

b. Higher energy consumption per unit product mass is a result of longer cycle times

c. A reduction in the overall solids (and liquid filtrate) productivity rate

d. An increase in the total volume of wash liquor consumed, which is particularly important when the wash is regenerated thermally for re-use, as would be the case with a solvent wash

e. A reduction in the filtration area required for the separation.

Of these factors (a)-(d) tend to increase separator operating costs (or decrease revenues in the case of (c)), whilst (e) reduces both capital and to some extent maintenance costs.

By repeated use of the simulation for a given cycle configuration it is possible to identify the optimum cake thickness (assuming that an optimum exists) to be formed during the filtration phase(s). The optimum is application specific and a compromise between the efficiency of cycle operations and the overall economics and time scale of the filter cycle operation. It is also clear that the effects of other process parameters on filter performance can also be readily assessed using the simulation.

\section{CONCLUSIONS}

This article outlines the methodology behind the simulation of batch filter cycles, using the diaphragm filter press as an example. It indicates how computer simulations can significantly improve the understanding of the operations that can occur during the filter cycle. Each of the theories used to model the cake formation, consolidation, deliquoring and washing phases has been proven in pilot scale and industrial tests, although they are capable of improvement from a fundamental point of view. The modelling procedures adopted for the simulation of the diaphragm filter press can be readily adapted to include optimisation routines and extended to encompass other types of separation equipment. When fully developed, such computer software should provide a suite of programs which are sufficiently powerful to minimise the number of costly and time consuming experiments which need to be performed to assess the suitability of equipment for a particular duty.

\section{NOMENCLATURE}

A total area of filter medium $\left(\mathrm{m}^{2}\right)$

$A_{f} \quad$ filtration area $\left(\mathrm{m}^{2}\right)$

$A_{f 0} \quad$ filtration area of a cylindrical filter element $\left(\mathrm{m}^{2}\right)$

$b \quad$ coefficients in eqn. (4) (-)

$C_{c} \quad$ modified consolidation coefficient $\left(\mathrm{m}^{2} \mathrm{~s}^{-1}\right)$

$C$ effective feed suspension concentration $\left(\mathrm{kg} \mathrm{m}^{-3}\right)$

$D$ molecular diffusivity of solute $\left(\mathrm{m}^{2} \mathrm{~s}^{-1}\right)$

$D_{L} \quad$ dispersion coefficient $\left(\mathrm{m}^{2} \mathrm{~s}^{-1}\right)$

$D_{n} \quad$ dispersion number (-)

e voids ratio (-)

$F \quad$ fraction of recoverable solute removed from filter cake (-)

Cite paper as: Wakeman R.J. and Tarleton E.S., 1999, Modelling and simulation of batch pressure filter cycles, Filtration and Separation, 36(8), 24-31. DOI: 10.1016/S0015-1882(99)80235-3. Also presented at Software for Filtration and Separation Conference, 1999, The Filtration Society, Birmingham, UK. 


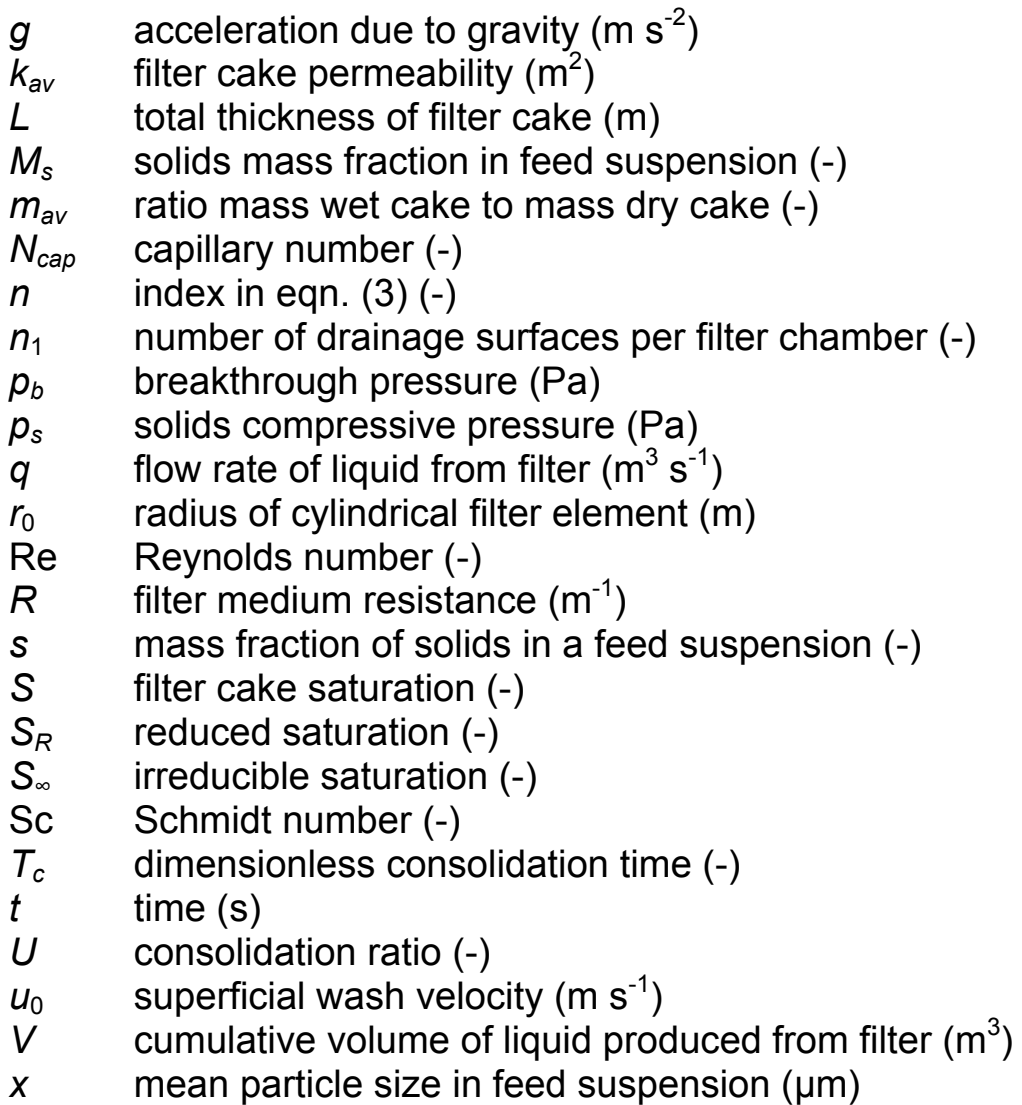

Greek symbols
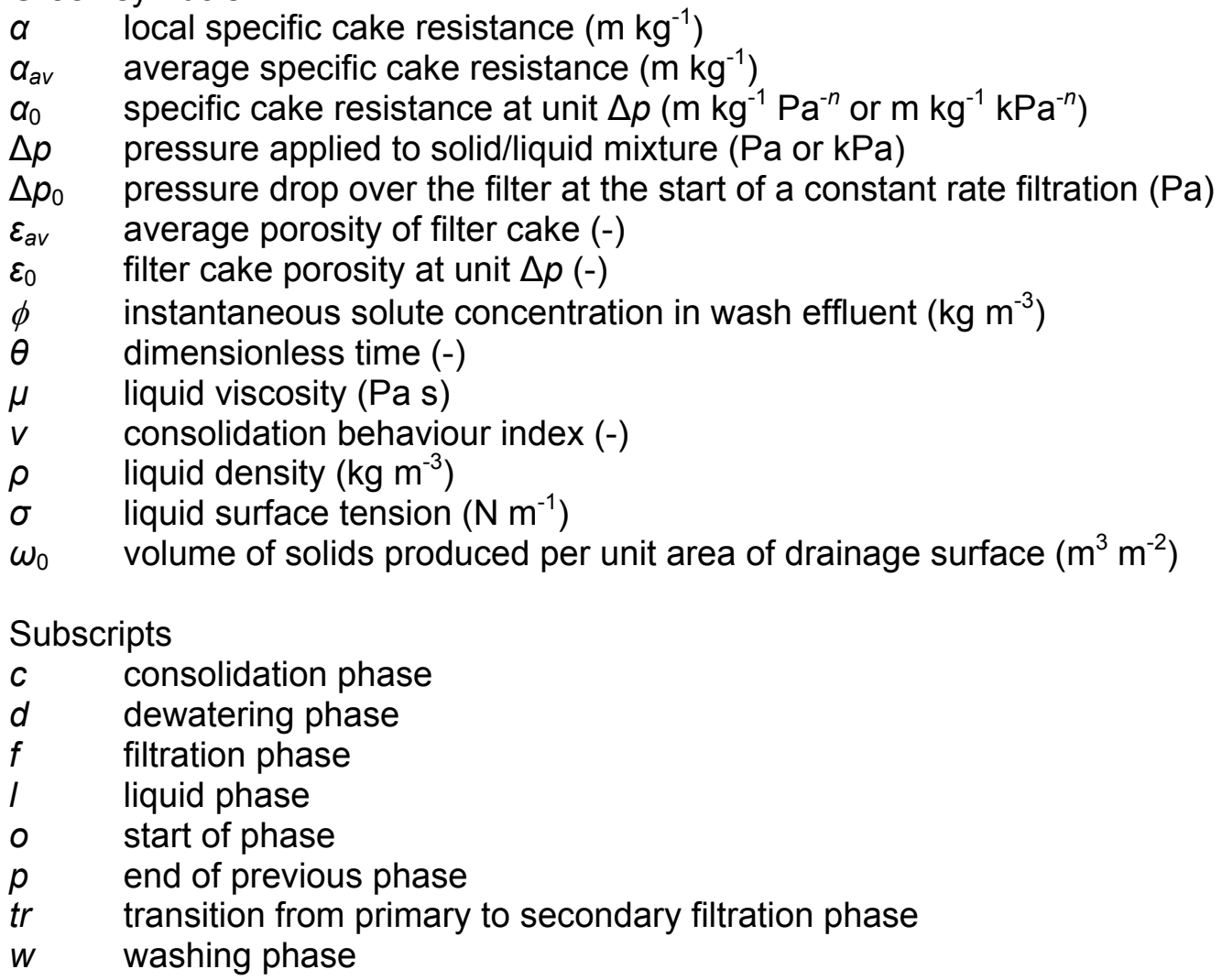
at equilibrium

\section{REFERENCES}

1. Tarleton E.S. and Wakeman R.J., 1991, Solid/Liquid Separation Equipment Simulation \& Design: $p^{C}$-SELECT - Personal Computer Software for the Analysis of Filtration and Sedimentation Test Data and the Selection of Solid/Liquid Separation Equipment, Separations Technology Associates.

2. Wakeman R.J. and Tarleton E.S., 1991, Solid/liquid separation equipment simulation and design an expert systems approach, Filtration and Separation, 28(4), 268.

3. Wakeman R.J., 1995, Selection of equipment for solid/liquid separation processes, Filtration and Separation, 32(4), 337.

4. Wakeman R.J. and Tarleton E.S., 1999, Filtration: Equipment Selection, Modelling and Process Simulation, xxvi+446, Elsevier, Oxford.

5. Wakeman R.J., Sabri M.N. and Tarleton E.S., 1991, Factors affecting the formation and properties of wet compacts, Powder Technology, 65(1-3), 283.

6. Buscall R. and White L.R., 1987, The consolidation of concentrated suspensions, J. Chem. Soc. Faraday Trans 1, 83, 873.

7. Landman K.A., Sirakoff C. and White L.R., 1991, Dewatering of flocculated suspensions by pressure filtration, Phys. Fluids A, 3(6), 1495.

8. Koenders M.A. and Wakeman R.J., 1996, The initial stages of the compact formation from suspensions by filtration, Chem. Eng. Sci., 51, 3897.

9. Koenders M.A. and Wakeman R.J., 1997, Filter cake formation from structured suspensions, Trans IChemE, 75(A), 309.

10. Koenders M.A. and Wakeman R.J., 1997, Initial deposition of interacting particles by filtration of dilute suspensions, AIChEJ, 43, 946.

11. Young I.M., in Solid/liquid Separation Equipment Scale-up, $2^{\text {nd }}$ Edn., 1986, D.B. Purchas and R.J. Wakeman (Eds.), pp.446-484, Uplands Press and Filtration Specialists, London.

12. Tiller F.M. and Leu W.F., 1980, Basic data fitting in filtration, J. Chinese Institute Engineers, $11,61$.

13. Shirato M., Murase T., Iritani E., Tiller F.M. and Alciatore A.F., 1987, Filtration in the Chemical Process Industry, in Filtration, M.J. Matteson and C. Orr (Eds.), Marcel Dekker, New York.

14. Wakeman R.J., 1976, Vacuum dewatering and residual saturation of incompressible filter cakes, International J. Mineral Processing, 3, 193.

15. Wakeman R.J., 1982, An improved analysis for the forced gas deliquoring of filter cakes and porous media, J. Separation Process Technology, 3, 32. 
16. Wakeman R.J., 1979, The performance of filtration post-treatment processes: 1. The prediction and calculation of cake dewatering characteristics, Filtration and Separation, 16(6), 655.

17. Wakeman R.J. and Tarleton E.S., 1990, Modelling, simulation and process design of the filter cycle, Filtration and Separation, 27(6), 412.

18. Wakeman R.J., 1980, The performance of filtration post-treatment processes: 2 . The estimation of cake washing characteristics, Filtration and Separation, 17(4), 67.

19. Wakeman, 1986, Transport equations for the filter cake washing, Chemical Engineering Research and Design, 64, 308.

20. Wakeman R.J. and Attwood G.J., 1988, Developments in the applications of cake washing theory, Filtration and Separation, 25, 272.

21. Wakeman R.J. and Attwood G.J., 1990, Simulations of dispersion phenomena in filter cake washing, Chemical Engineering Research and Design, 68, 161. 


\section{FIGURES AND TABLES}

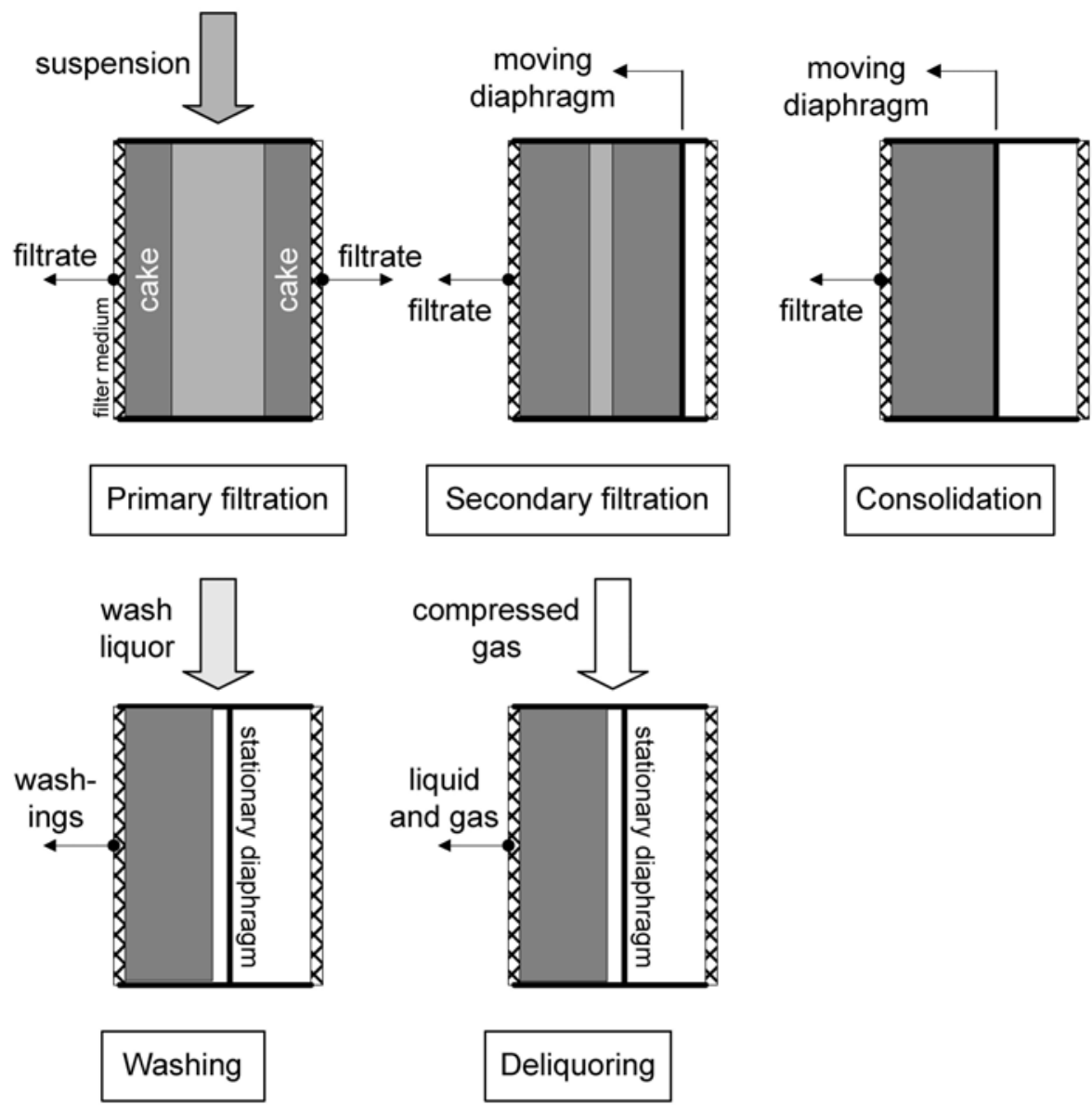

Figure 1: Operations which can be carried out during a diaphragm filter press cycle. 

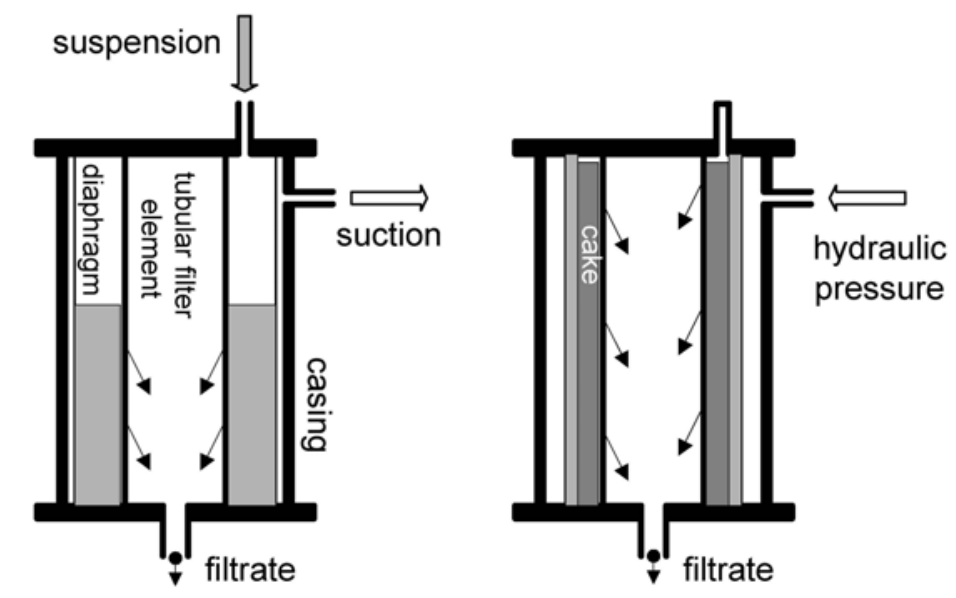

Filling with suspension

Filtration and consolidation (via diaphragm)
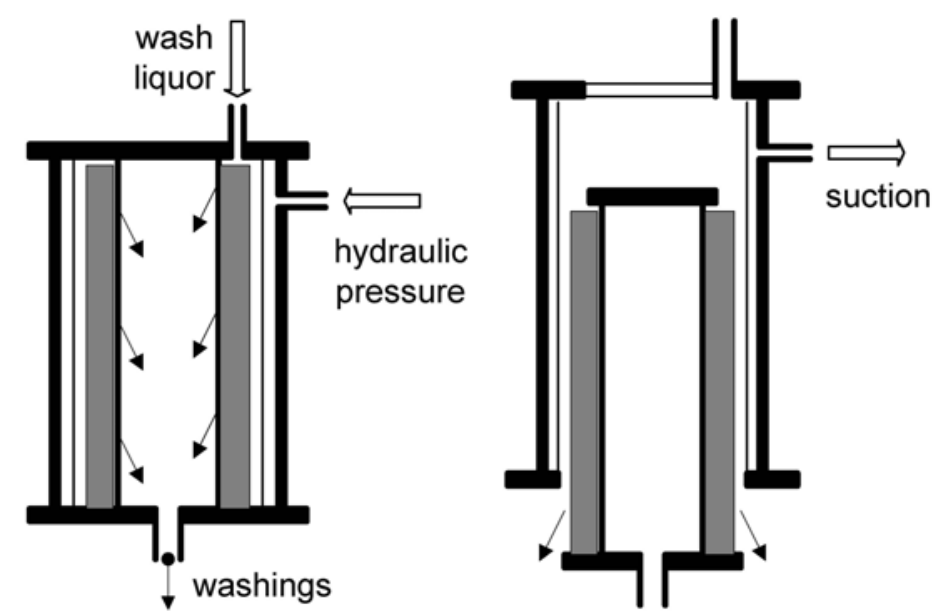

Washing (via diaphragm)

Cake discharge

Figure 2: Schematic diagram of a tube press cycle. 


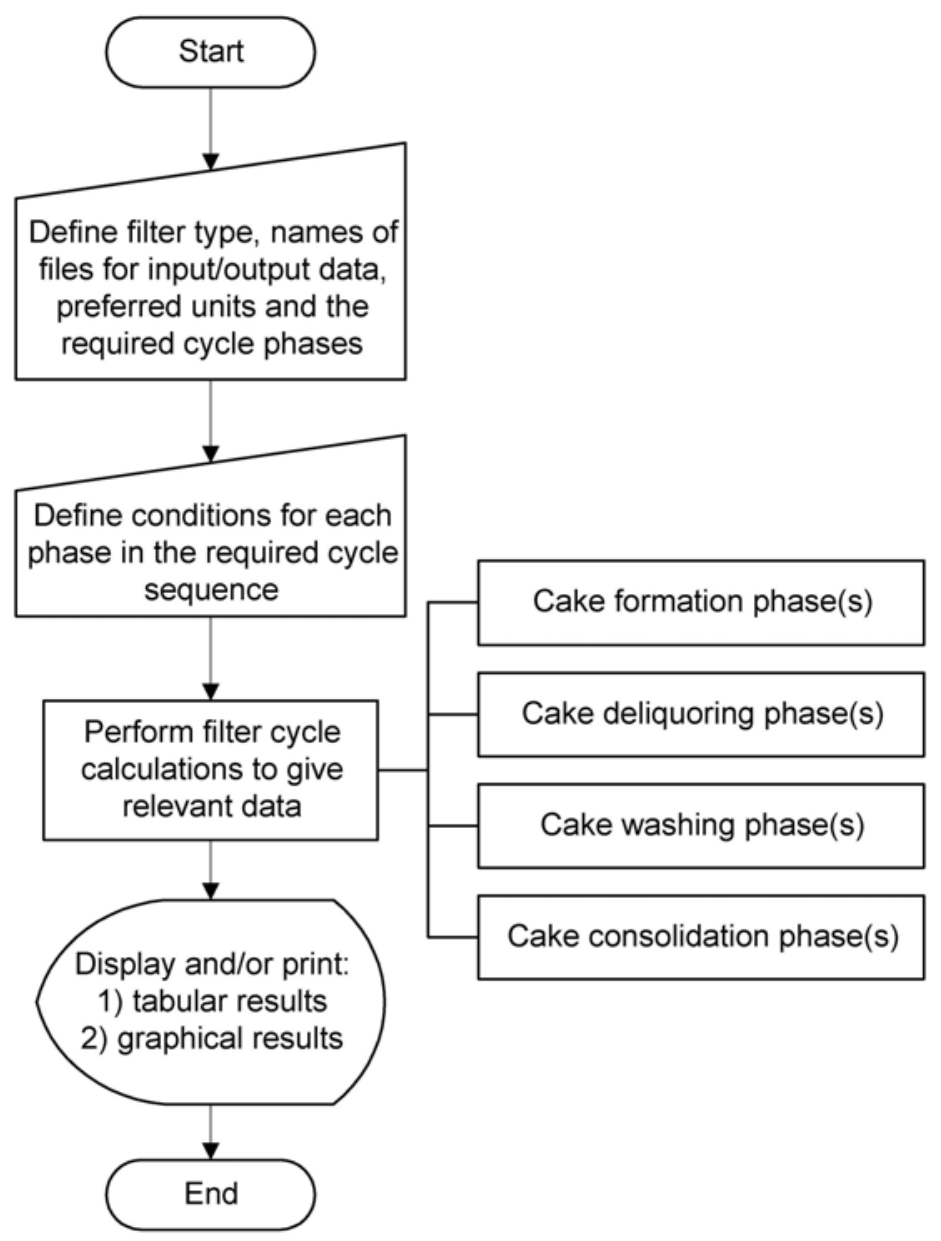

Figure 3: Basic flowsheet for filter cycle simulations. 


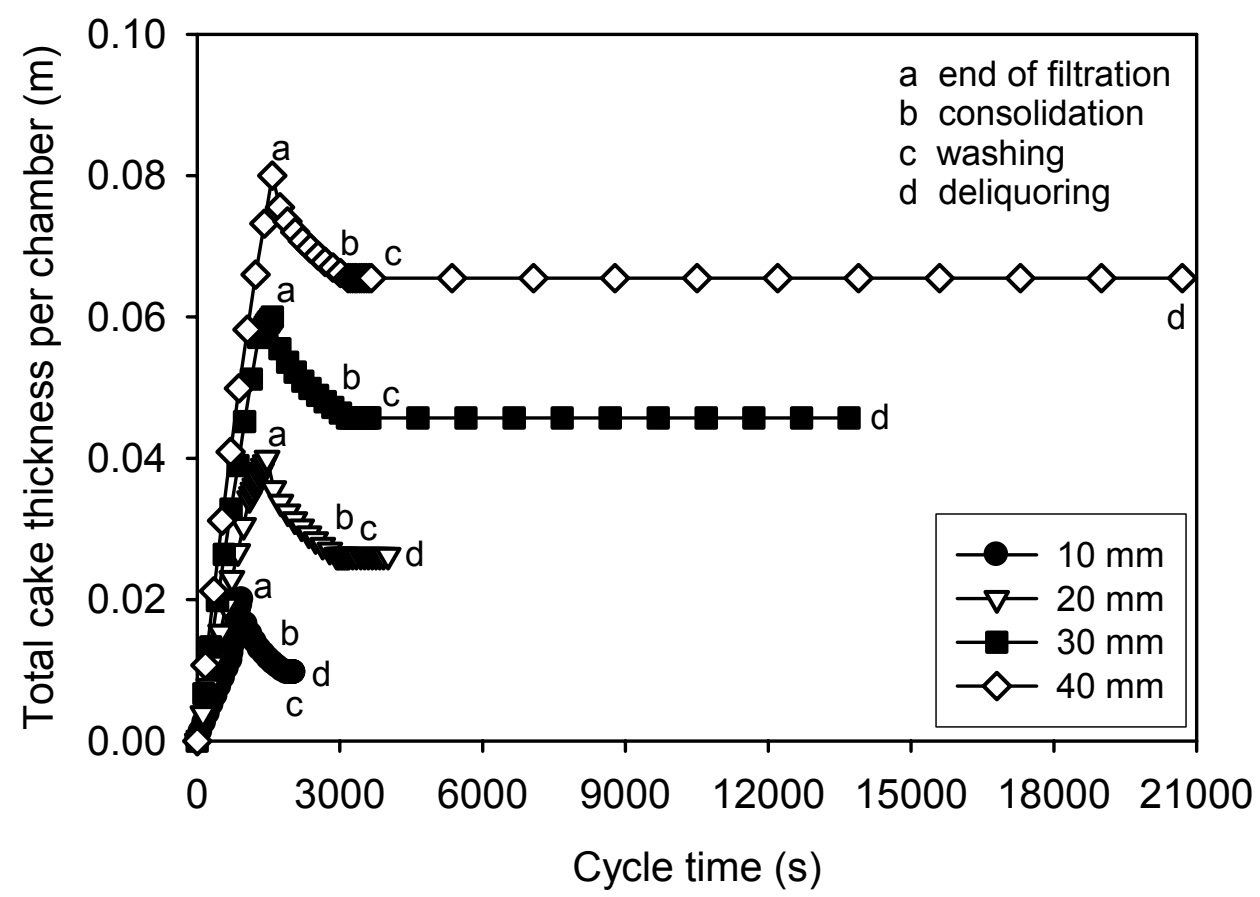

Figure 4: Variation of total cake thickness formed in a chamber during the operating cycle of the diaphragm filter press.

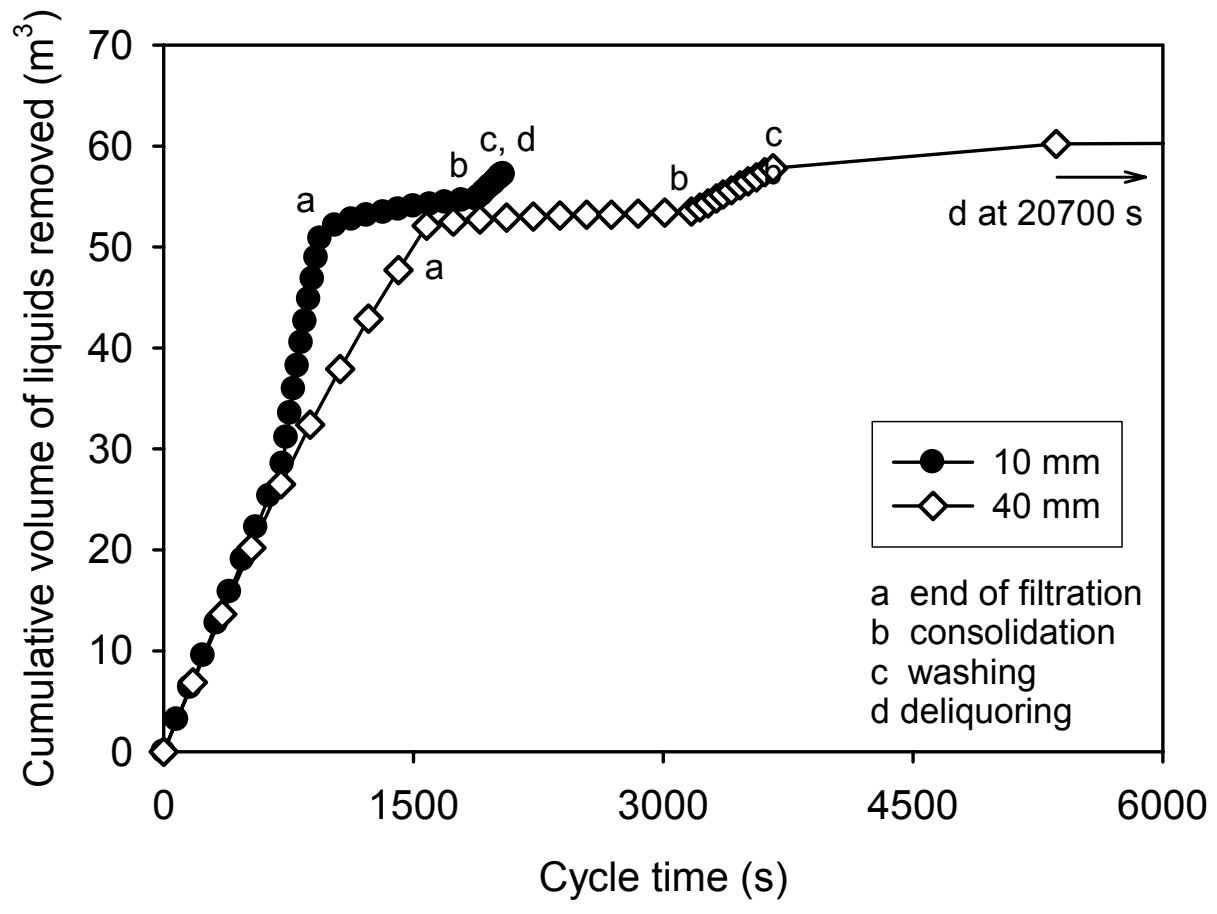

Figure 5: Effect of formed cake thickness on the volume of liquids extracted during a diaphragm filter press cycle. 


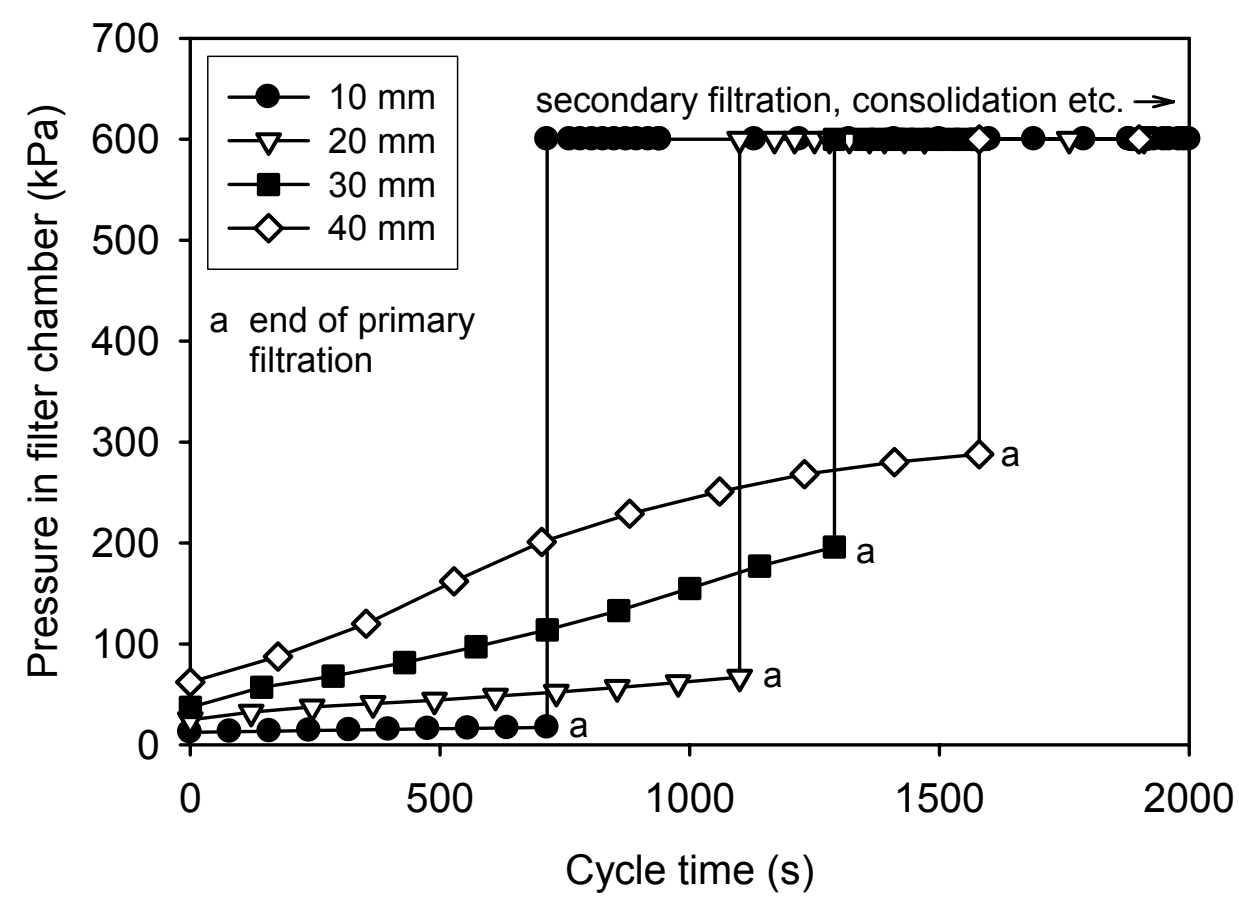

Figure 6: Effects of formed cake thickness on the pressures generated in the filter chambers of the diaphragm filter press.

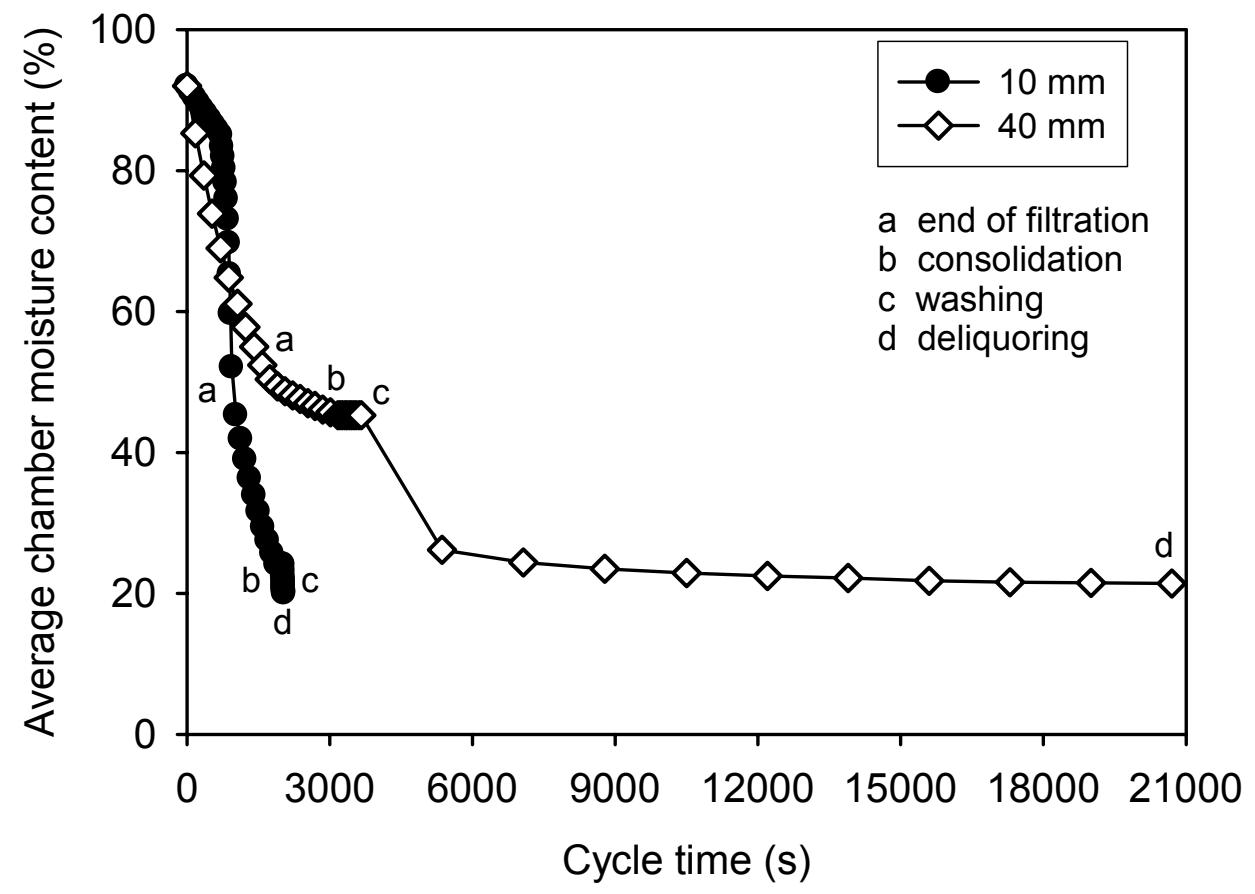

Figure 7: Influences of formed cake thickness on average chamber moisture content during a diaphragm filter press cycle. 


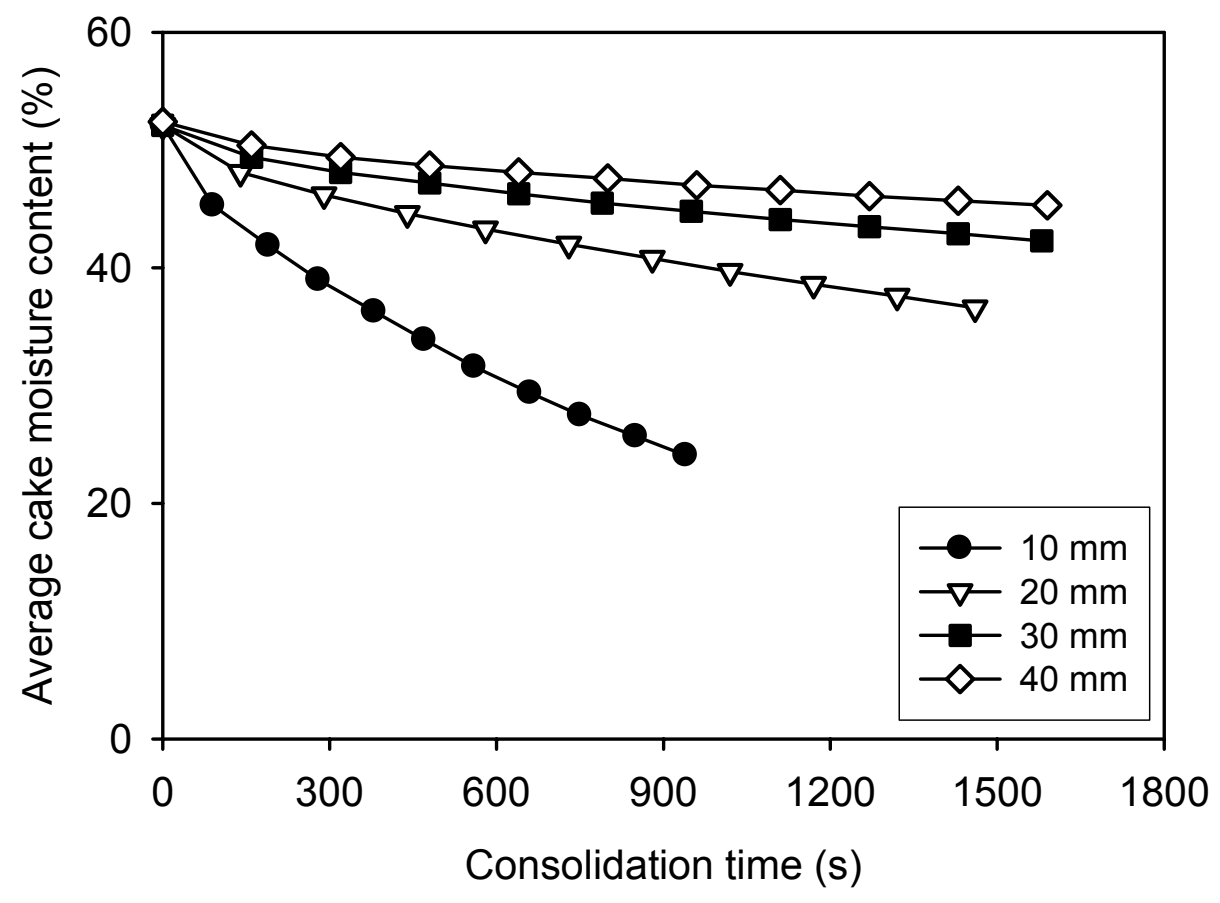

Figure 8: Influence of formed cake thickness on the consolidation process in a diaphragm filter press cycle.

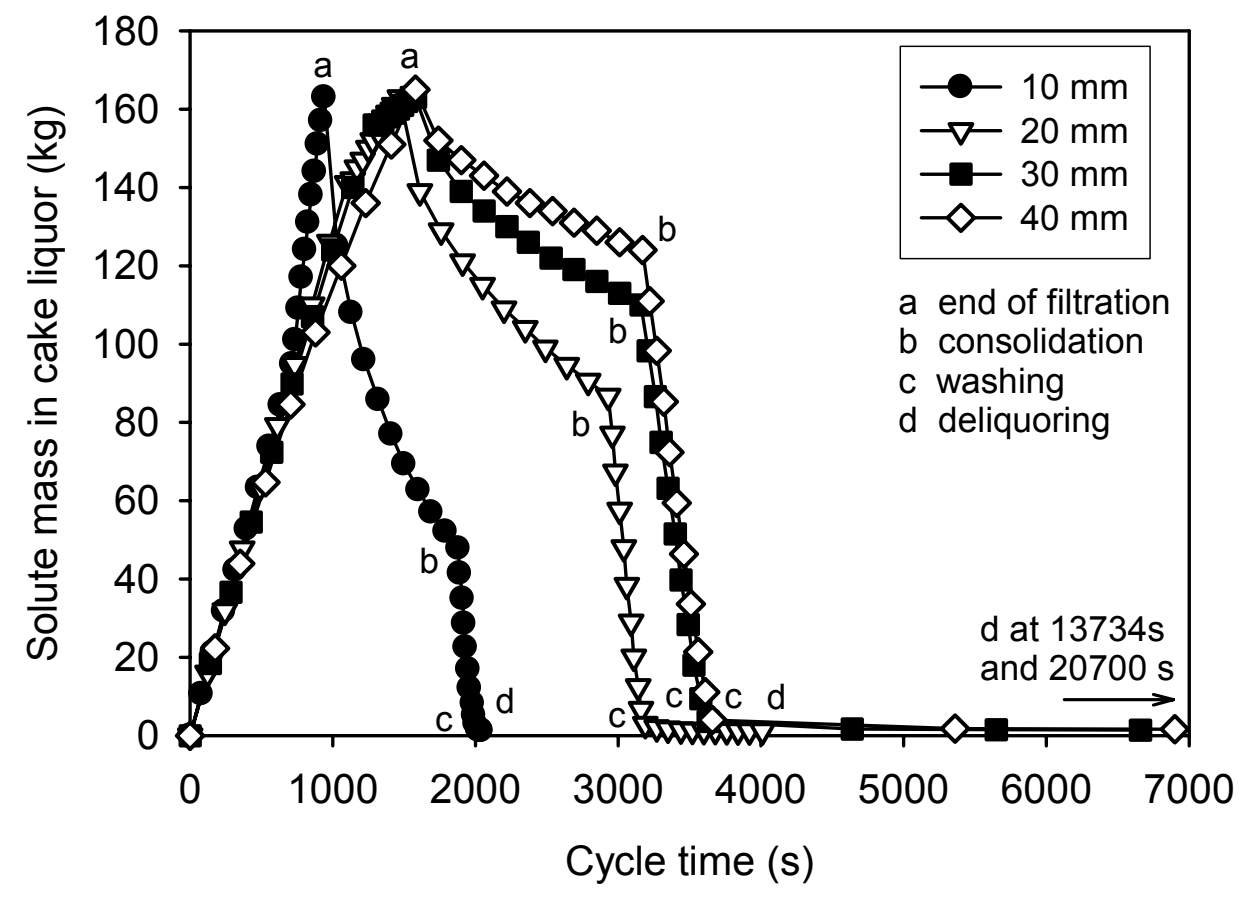

Figure 9: Effects of cake thickness on the total mass of solute in the filter cakes formed during the diaphragm filter press cycle. 


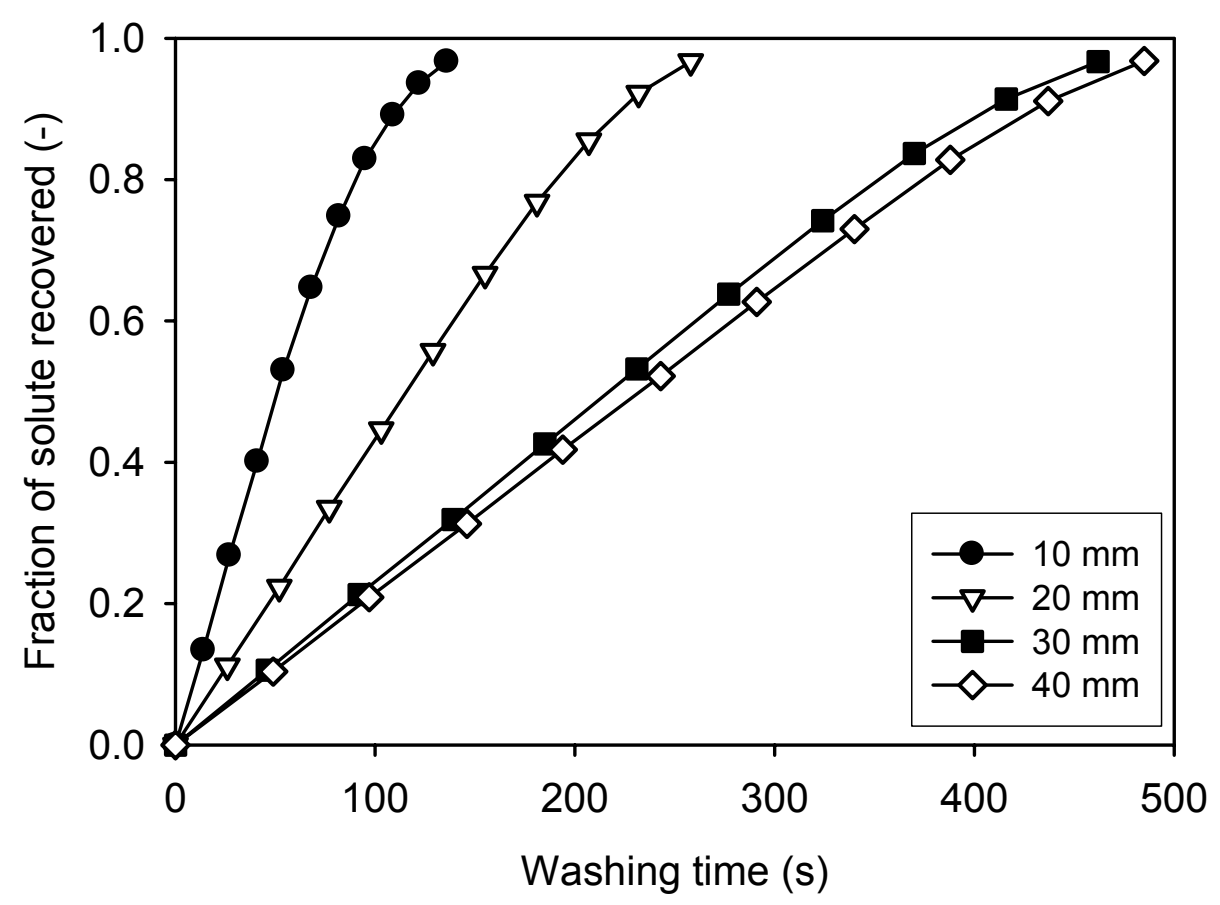

Figure 10: Effects of formed cake thickness on the fractional recovery of solute during the washing phase of a diaphragm filter press cycle.

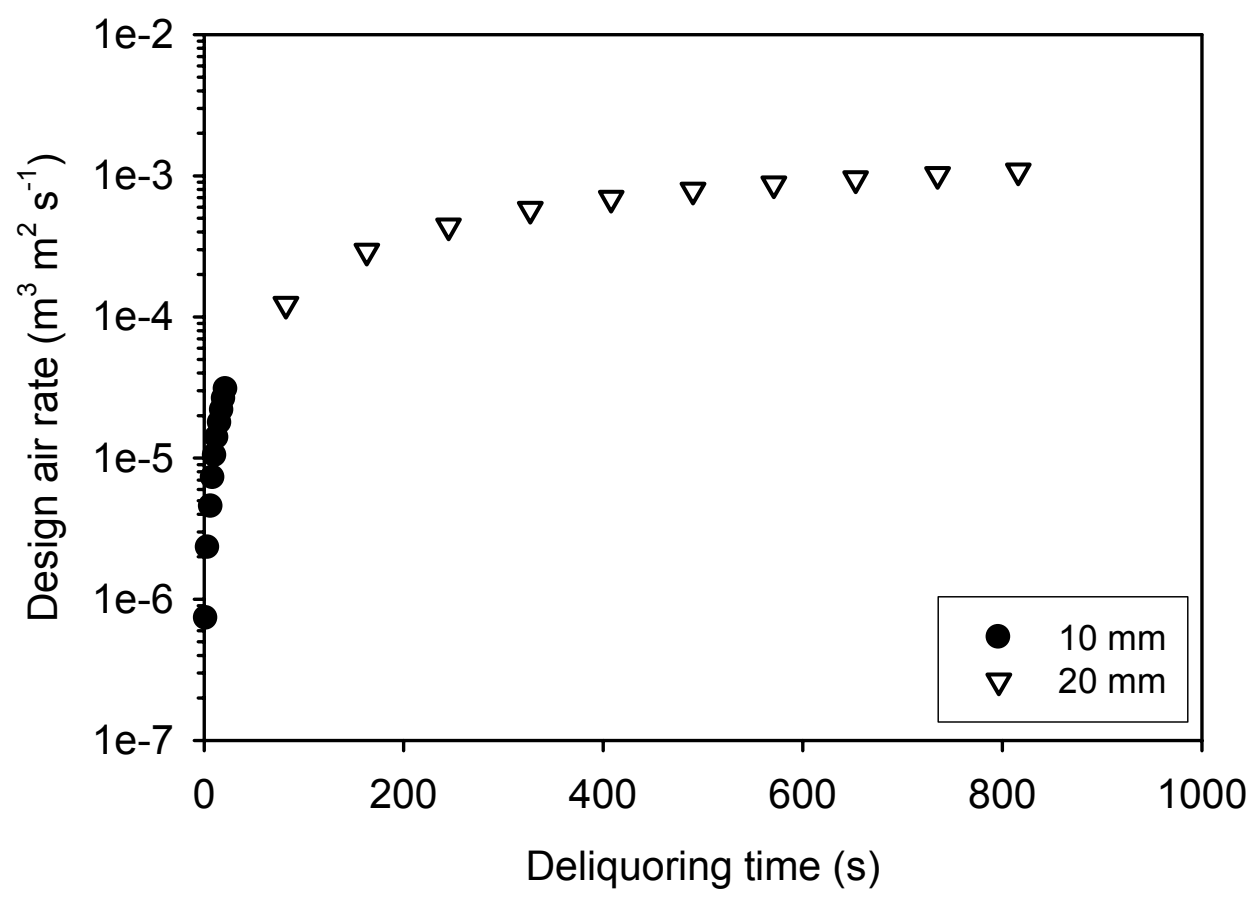

Figure 11: Effects of formed cake thickness on the air rate required to deliquor the cakes formed in the diaphragm filter press cycle. 


\begin{tabular}{|c|c|}
\hline Filtrate volume - Filtration time relationships & Cake thickness in filter \\
\hline $\begin{array}{l}\text { Nutsche, diaphragm and filter presses: Constant pressure } \\
\frac{t_{f}}{V_{f}}=\frac{\alpha_{a v} c \mu_{l}}{2 A_{f}^{2} \Delta p_{f}} V_{f}+\frac{\mu_{l} R}{A_{f} \Delta p_{f}}\end{array}$ & $L_{f}=\frac{V_{f}}{A_{f}} \frac{s\left(\rho_{s}\left(m_{a v}-1\right)+\rho_{l}\right)}{\rho_{s}\left(1-m_{a v} s\right)}$ \\
\hline 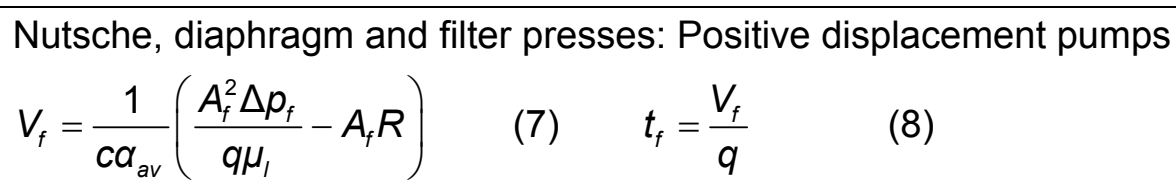 & As above \\
\hline $\begin{array}{l}\text { Nutsche, diaphragm and filter presses: Centrifugal pumps } \\
V_{f}=\frac{1}{c a_{a v}}\left(\frac{A_{f}^{2} \Delta p_{f}}{q \mu_{l}}-A_{f} R\right) \\
q \text { is given by the pump curve }\end{array}$ & As above \\
\hline $\begin{array}{l}\text { Tube press: Constant pressure } \\
\frac{t_{f}}{V_{f}}=\left(\frac{K_{1}}{2} V_{f}+K_{2}\right)+\frac{2 c}{A_{f 0} r_{0} \rho_{c}}\left(\frac{K_{1}}{3} V_{f}+\frac{K_{2}}{2}\right) V_{f} \\
A_{f 0} \text { is filter area at start of filtration; } r_{0} \text { is filter element radius } \\
K_{1}=\frac{a_{a v} c \mu_{1}}{A_{f 0}^{2} \Delta p_{f}} \quad K_{2}=\frac{\mu_{1} R}{A_{f 0} \Delta p_{f}}\end{array}$ & $L_{f}=r_{0}\left(\sqrt{1+\frac{2 c V_{f}}{A_{f 0} r_{0} \rho_{c}}-1}\right)$ \\
\hline $\begin{array}{l}\text { Tube press: Positive displacement pumps } \\
V_{f}=q t_{f} \\
t_{f}=\frac{A_{f 0} r_{0} \rho_{c}}{2 c q}\left(\exp \left(\frac{2 A_{f 0}\left(\Delta p_{f}-\Delta p_{0}\right)}{q \mu_{l} \alpha_{a v} r_{0} \rho_{c}}\right)-1\right)\end{array}$ & As above \\
\hline $\begin{array}{l}\text { Tube press: Centrifugal pumps } \\
V_{f}=\frac{1}{c a_{a v}}\left(\frac{A_{f}^{2} \Delta p_{f}}{q \mu_{l}}-A_{f} R\right) \\
\text { where } A_{f}=A_{f 0} \sqrt{1+\frac{2 c V_{f}}{r_{0} A_{f 0} \rho_{c}}} \\
t_{f}=\int_{0}^{v} \frac{d V_{f}}{q}\end{array}$ & As above \\
\hline
\end{tabular}

Table 1: Working equations to simulate the cake formation stages in the filter cycle. 


\begin{tabular}{|c|c|}
\hline Flow rate $\left(\mathrm{m}^{3} \mathrm{~s}^{-1}\right)$ & Pressure $(\mathrm{kPa})$ \\
\hline 0 & 360 \\
0.022 & 300 \\
0.03 & 250 \\
0.035 & 200 \\
0.037 & 150 \\
0.0385 & 100 \\
0.0395 & 50 \\
0.04 & 0 \\
\hline
\end{tabular}

Table 2: Pump characteristics. 


\begin{tabular}{|l|l|}
\hline Parameter & Value \\
\hline Filter and septum characteristics & \\
Filter chamber thickness $(T)$ & $80 \mathrm{~mm}$ \\
Filter medium resistance $(R)$ & $3 \times 10^{11} \mathrm{~m}^{-1}$ \\
& \\
Operating conditions & $600 \mathrm{kPa}$ \\
Secondary filtration, consolidation and & \\
washing pressures $\left(\Delta p_{f}, \Delta p_{c}\right.$ and $\left.\Delta p_{w}\right)$ & $650 \mathrm{kPa}$ \\
Deliquoring pressure $\left(\Delta p_{d}\right)$ & $100 \mathrm{kPa}$ \\
Barometric pressure $\left(p_{B}\right)$ & 0.08 \\
Solids mass fraction in the feed $(s)$ & $30 \mathrm{~kg} \mathrm{~m}^{-3}$ \\
Solute concentration in the feed $\left(\phi_{0}\right)$ & $0.5 \mathrm{~kg} \mathrm{~m}^{-3}$ \\
Solute concentration in the wash $\left(\phi_{w}\right)$ & \\
& \\
Cake properties & $20 \mathrm{~mm}$ \\
Max. thickness on each filter surface & $\alpha_{a v}=2.4 \times 10^{9} \Delta p^{0.6} \mathrm{~m} \mathrm{~kg}^{-1}$ \\
Constitutive equations for the & $\varepsilon_{a v}=0.75 \Delta p^{-0.0039}$ \\
filtration phase $(\mathrm{s}), \Delta p$ in $\mathrm{kPa}$ & $C_{c}=1 \times 10^{-8} \Delta p^{0.1} \mathrm{~m}^{2} \mathrm{~s}^{-1}$ \\
Constitutive equations for the & $\varepsilon_{\mathrm{av}}=0.62 \Delta p^{-0.12}$ \\
consolidation phase, $\Delta p$ in $\mathrm{kPa}$ & 3 \\
Consolidation behaviour index $(v)$ & \\
Particle and fluid properties & $10 \mu \mathrm{m}$ \\
Mean size of solids $\left(x_{a v}\right)$ & $2500 \mathrm{~kg} \mathrm{~m}^{-3}$ \\
Density of solids $\left(\rho_{s}\right)$ & $998 \mathrm{~kg} \mathrm{~m}^{-3}$ \\
Density of filtrate and wash $\left(\rho_{l}\right)$ & $0.001 \mathrm{~Pa} \mathrm{~s}^{\prime}$ \\
Viscosity of filtrate and wash $\left(\mu_{l}\right)$ & $0.07 \mathrm{~N} \mathrm{~m}^{-1}$ \\
Surface tension of filtrate and wash $(\sigma)$ & 0.26 \\
Irreducible cake saturation $\left(S_{\infty}\right)$ & $1.8 \times 10^{-5} \mathrm{~Pa} \mathrm{~s}^{-1}$ \\
Viscosity of air $\left(\mu_{a}\right)$ & $1 \times 10^{-9} \mathrm{~m}^{2} \mathrm{~s}^{-1}$ \\
Solute diffusivity $(D)$ & \\
\hline
\end{tabular}

Table 3: Operating conditions and filtration parameters of the filter to be simulated. 


\begin{tabular}{|c|c|c|c|}
\hline $\begin{array}{c}\text { Cake thickness per } \\
\text { chamber }(\mathrm{mm})\end{array}$ & $\begin{array}{c}\text { Total filtration } \\
\text { area }\left(\mathrm{m}^{2}\right)\end{array}$ & $\begin{array}{c}\text { Total cycle } \\
\text { time }(\mathrm{h})^{\dagger}\end{array}$ & $\begin{array}{c}\text { Nominal solids } \\
\text { production rate }\left(\mathrm{kg} \mathrm{h}^{-1}\right)^{\dagger}\end{array}$ \\
\hline $20(2 \times 10)$ & 744 & 0.6 & 8772 \\
$40(2 \times 20)$ & 373 & 1.1 & 4545 \\
$60(2 \times 30)$ & 248 & 3.8 & 1316 \\
$80(2 \times 40)$ & 188 & 5.8 & 862 \\
\hline
\end{tabular}

tignores cake discharge time, cloth cleaning time etc.

Table 4: Effects of cake thickness on filter size and productivity. 